\title{
EVALUATION OF THE PRE-DETECTIVE SIGNAL PRIORITY FOR BUS RAPID TRANSIT: COORDINATING THE PRIMARY AND SECONDARY INTERSECTIONS
}

\author{
Min Yang ${ }^{1}$, Gang Sun ${ }^{2}$, Wei Wang ${ }^{1}$, Xin Sun ${ }^{3}$, Jian Ding ${ }^{1}$, Jing Han ${ }^{1}$ \\ ${ }^{1}$ School of Transportation, Southeast University, China \\ ${ }^{2}$ School of Transportation Engineering, Tongji University, China \\ ${ }^{3}$ Dept of Civil Engineering, Texas A\&M University, United States
}

Submitted 10 March 2013; resubmitted 9 July 2013, 3 November 2013; accepted 19 February 2014; published online 28 January 2015

\begin{abstract}
Since the traditional transit priority strategy can only adjust signal timing in a limited range and is not suitable for all kinds of signal timing designs, it cannot provide enough priority for Bus Rapid Transit (BRT). In addition, traditional transit priority strategy has caused serious interferences with other traffic. This study proposes a pre-detective signal priority strategy for BRT with coordination between primary and secondary intersections. By pre-detecting, the time buses arrive at the primary intersection, the signal timing of both the primary and secondary intersections, along with the offsets, are adjusted simultaneously, based on the common length and the green ratio of each phase. In this method, the signal cycle constraints are clarified, and the bus control coordination between intersections has been taken into consideration. In this paper, one direction traffic is taken as a study example to testify the effectiveness of this method. The study uses the data collected from Changzhou, China, and a microscopic traffic simulation software PTV VISSIM with four simulation scenarios defined: no signal priority, traditional signal priority, pre-detective signal priority and pre-detective signal priority with coordination. This paper selects a set of indicators to evaluate the traffic operation for both public transit and private traffic. Results show that pre-detective signal priority with coordination is the most effective, with the total bus intersection delay decreases by $67.4 \%$ and the bus headway adherence declines by approximately $40 \%$ at all the primary and secondary stations of BRT line 1 . Moreover, the negative effects that could happen with providing signal priority for BRT, such as increasing the delay and length of queue of private traffic at the intersections, are significantly reduced.
\end{abstract}

Keywords: bus rapid transit; pre-detective signal priority; coordinated control; microscopic simulation; strategy.

\section{Introduction}

Bus Rapid Transit (BRT) is already in wide use all over the world. Some cities in China had already implanted their own BRT systems. However, compared with normal public transit, BRT's advanced transport facilities, especially the exclusive bus lanes which provide passage priority for buses, have significantly reduced bus travel delay, which is still comparatively high experienced by the passengers. Bus travel delay can be affected by many factors, such as the signal timing plan at intersections, road geometric design, traffic volume, traffic distribution and weather conditions, while, bus intersection delay is the most obvious among the whole travel delay. Shown in several surveys of BRT operation, bus intersection delay accounts for approximately $40-60 \%$ of the total delay (TRB 2003; Wang et al. 2010). Therefore, we focus our research on the optimization of BRT signal timing plan, using simulation to control other factors that can affect bus delay, to testify the significance of a good BRT's signal design to improving its service quality.

Early studies mainly focused on how to achieve a passive transit signal priority strategy. Eichler and Daganzo (2006), and Yagar (1993) analysed some variables, including saturation of single intersection, bus service frequency, bus proportion, passengers' behaviour at the bus station and others, and established the bus priority control target optimal model to realize the bus priority. Vasudevan (2005) used the continuous running control of private traffic as the constraint condition and the minimum of average delay as the optimization goal, and established the network coordinate controlled transit signal priority strategy model. However, owing to

Corresponding author: Min Yang

E-mail: yangmin@seu.edu.cn 
the flexible traffic flow change at intersections, the time resources of the intersections can be a problem because of the off-line passive transit signal priority strategy. In addition, the active transit signal priority strategy overcomes the shortcomings of the passive transit signal priority to a large extent.

Traditional features of active transit signal priority strategy are green signal extensions; early green recalls and phase inserts. Xu and Zheng (2009), Toledo et al. (2010) and Wu et al. (2012) added the average red time of pedestrian phases and early green request service rates into the evaluation indexes, and established the behaviour-based multipurpose traffic simulation model. They used the Global Position System (GPS) technique to analyse delays of whole bus lines, and determined the appropriate transit signal priority strategy. Traditional active transit signal priority strategy mainly controls the signal statically, thus making it hard to be applied to situations like phases changing abruptly and beyond adjustment allowance, which seriously disturbs other traffic. Koehler and Kraus (2010), Ekeila et al. (2009) and Liu et al. (2007) used real time data obtained by the GPS and Automatic Vehicle Location (AVL) techniques to forecast the travel time of the crossing and the queuing situation at the target intersection and adjusted the time to trigger the traffic signal for priority as soon as the time at which the bus left the stop. This method increased the efficiency of the active transit signal priority. Head (2006) proposed the predictive transit signal priority strategy, which extended the detection distance, and adjusted the length of each phase in a signal cycle proportionally, to give priority to the buses. Along with researches on predictive transit signal priority strategy, Wadjas and Furth (2003), and Langdon (2002) applied this technology to priority control for railway transit, which immensely expanded the scope of application. Based on the AVL data, Chen (2005) added the headway constraints into the signal priority algorithm. He used the method of inserting a special phase or skipping the phase, and comprehensively analysed the whole profit over all kinds of vehicles gained by offering signal priority to buses with conditions, while coordination and prediction are not the emphasis of his research.

Predictive transit signal priority strategy, as mentioned before, starts the bus detection much earlier before bus arrives and adjusts the length of each phase of signal timing proportionally so that the adjustment can be more sufficient and gentle. However, this method ignores the coordination between each intersection, thus can lead to an accumulative delay at the secondary intersection, causing the effectiveness and operation of the arterial to decrease significantly. Therefore, under the premise of existing exclusive BRT lanes, focusing on a single direction, this proposed pre-detective signal priority strategy, with coordination between the primary and secondary intersections, can maximize the efficiency of the transit system, while minimizing interferences caused to private traffic.

The remainder of this paper is organized as follows: an introduction of pre-detective signal priority using a coordination algorithm, description the PTV VISSIM simulation and the result analysis. Four simulation scenarios were set, because the traffic condition remains all the same under the four scenarios, the actual bus travel time was collected and used in the model. In order to testify the effectiveness and contribution of our proposed pre-detective method, the BRT line 1 in Changzhou was taken as a study example. The last part of this paper contains the conclusion and future directions.

\section{Methodology}

The flowchart in Fig. 1 illustrates the algorithm of the pre-detective signal priority strategy with coordination between the primary and secondary intersections.

Our control strategy makes its decision whether to give signal priority based on the bus travel data collected from the comparatively farther sensors upstream. Once decided to give signal priority, the detailed signal priority strategy for buses will be generated. The control method mainly consists of three parts, which are the calculation of the critical cycle length, the cycle length constraints and the coordination between adjacent intersections. The critical cycle length is determined by the ideal offset between the bus arrival time and green signal duration. The cycle length constraints are used to control the selection of different types of signal priority through the penalty factor and expected delay. The coordination between adjacent primary and secondary intersections makes it possible for the buses to travel smoothly and guarantee the success of bus priority.

\subsection{Algorithm of Adjusting Signal Timing}

There are two types of detectors needed in this signal priority strategy, which are the bus arrival detector and the bus departure detector. The first one is located at a travel time of 150 to 300 seconds ahead of the stop line upstream the primary intersection and the latter one is placed at the stop line of the primary intersection. Only when the current bus which is applying for signal priority passes through the stop line of the primary intersection, the arrival-detection system will be re-activated (except the first bus of the line) and will continue accepting applications for signal priority for the next bus.

Adjustment of the cycle length. The cycle here refers to the signal period of the main intersection and is used as the common cycle length in coordination between adjacent intersections. There are two types of adjustment for priority: one extends the signal phase length, and the other compresses it. The adjustment process is shown in Fig. $2 \mathrm{a}, \mathrm{b}$. If the bus reaches the stop line in the green phase of its direction, there is no need to adjust the signal timing.

When signal priority is needed, the ideal time difference $\Delta t$ between the predicted arrival time and the ideal arrival time of a bus reaching the stop line of the primary intersection should be calculated for both the extended-phase and compressed-phase adjustments ( $\Delta t_{\text {ext }}$ stands for the total time difference in extendedphase adjustment, and $\Delta t_{c o m}$ is used in the compressedphase method). The number of adjustable cycles $N$ should also be calculated separately for both adjustment 


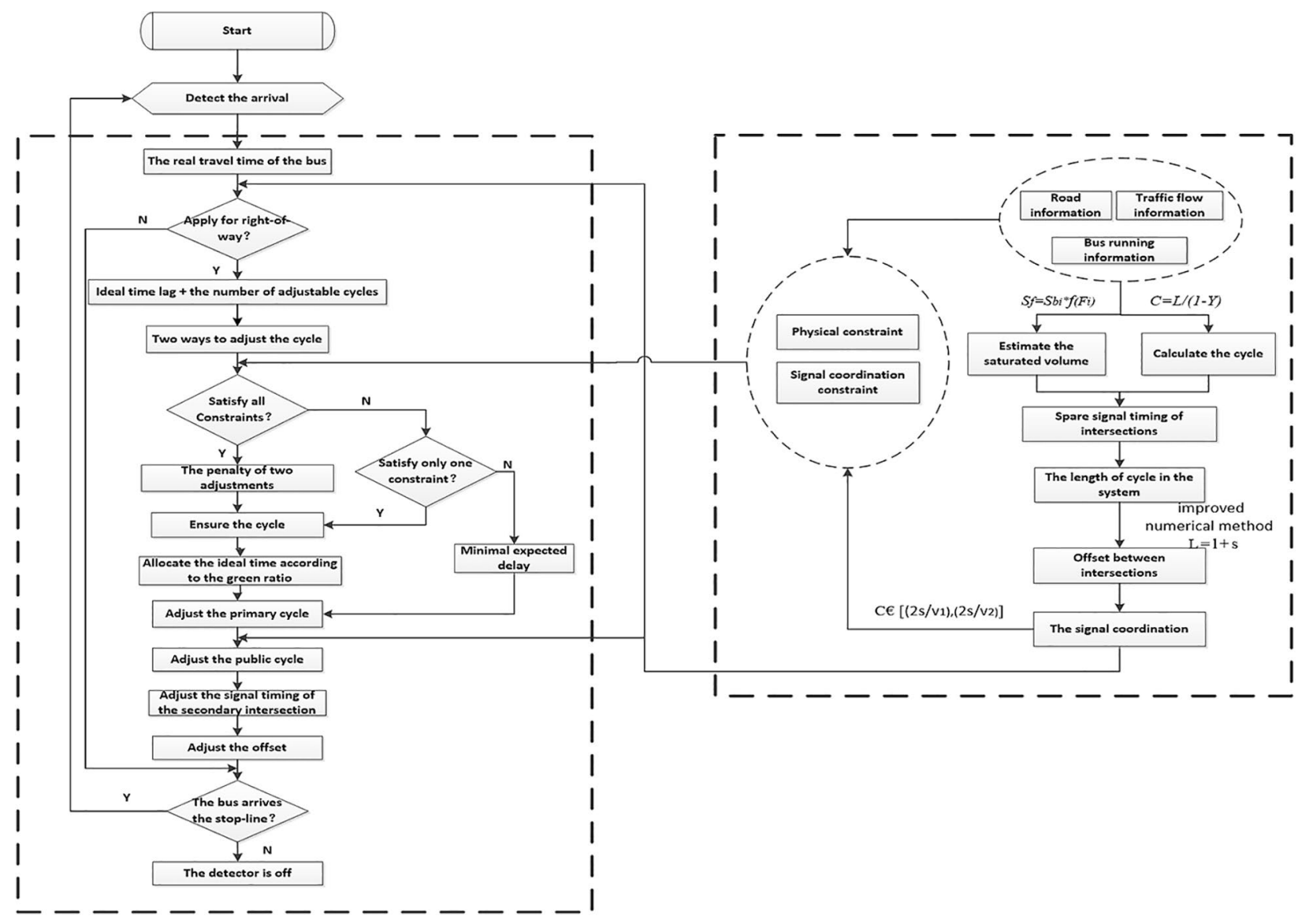

Fig. 1. Flowchart of the pre-detective signal priority strategy with coordination

a)

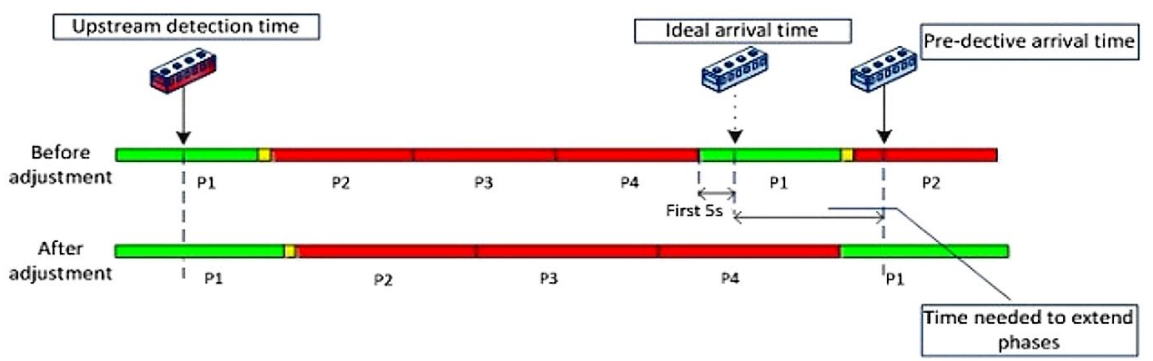

b)

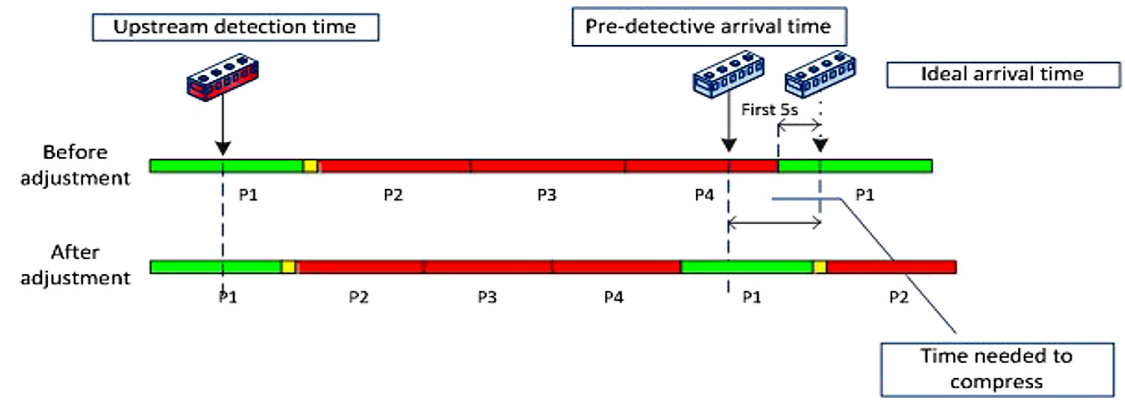

c)

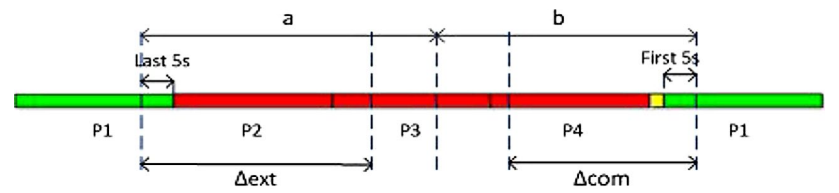

Fig. 2. Extension option (a), compression option (b) and sketch map of minimal expected delay (c) 
methods. Then, the specific strategy will be chosen according to penalty factor $P$ (for extended-phase adjustment: $P=\Delta t_{\text {ext }}+0.025 \cdot \Delta t_{\text {ext }}^{2}$ and for compressed-phase method: $P=\Delta t_{\text {com }}$ ) and expected delay $D$ (for extendedphase adjustment: $D=a-\Delta t_{\text {ext }}$; for compressed-phase method: $\left.D=b-\Delta t_{\text {com }}\right)-$ see Fig. 2 c. The parameter $a$ stands for the time difference between the predicted bus arrival time and the last 5 seconds of the bus service phase in the cycle. The parameter $b$ is the time difference between the predicted bus arrival time and the first 5 seconds of the bus service phase in the cycle which follows the cycle that a bus arrives and the bus's arrival time.

Once the adjustment method is determined, $\Delta t$ should be distributed to the signal phases of every adjustable cycle, according to the green ratio of each phase:

$$
\Delta t_{i}=\frac{\Delta t \cdot \lambda_{i}}{N},
$$

where: $\Delta t_{i}$ represents the value of the adjustment of the $i$ th phase in the adjustable cycle; $\Delta t$ stands for the ideal time difference; $\lambda_{i}$ is the green ratio of $i$ th phase in the cycle of the subject intersection.

The adjustment of the cycle length of the secondary intersection equals to the adjusted cycle length of the primary intersection, shown in Eq. (1), and the adjustment is carried out simultaneously with the adjustment of the primary intersection.

When one priority application of the primary intersection is completed and there is no more coming bus according to the detectors, the signal timing of the intersection should be restored to the original 'optimal' settings, which is based on minimizing the time loss for private traffic.

Signal cycle constraints. Inappropriate signal timing could create obstacles for left-turning vehicles when entering left-turn lanes, thus could result in the decreasing of the efficiency of both left-turning and right-turning vehicles. To prevent the queue from overflowing, the intersection signal design should satisfy the maximum signal cycle constraints. Moreover, on the basis of coordination, it is better to keep a relatively high operation speed for the whole coordinating system. The high speed can improve the efficiency and reliability of bus operation, while the private traffic can also be benefited. In this paper, the coordination constraints are based on the improved numerical method used on arterial coordination. The minimal and maximal signal length of both the primary and secondary intersections are calculated as twice the ideal space between the signal locations $s$ divided by the lower limit $v_{1}$ or the upper limit $v_{2}$ of the bus system speed.

\subsection{Coordinated Control between Intersections}

Numerical methods are commonly used to calculate the offset in signal timing design. This section explains the differences between the improved numerical method and the traditional one.

In contrast to private traffic, which can travel freely between two intersections, (neglecting the internal and external factors of the traffic flow), buses must pull in and out of stations and stop at the station for board- ing or alighting passengers. Thus, buses have different travel time patterns with private traffic when driving on the same section. In this study, we will transfer the stop delays of the buses, $t$, into an indicator of distance, $s=$ $v \cdot t$ ( $s$ plays an important role in deciding the location of detectors and the number of stops of the buses investigated). According to the corrected distance $L=l+s$ (where $l$ means the actual space distance), the percentage of offset between two intersections will be redefined using the arterial coordination control algorithm. Additionally, the offset used for coordination between two intersections should be adjusted considering the revised common cycle length.

\subsection{PTV VISSIM Simulation Model}

To show the traffic operation characteristics under different signal conditions, we applied simulation to our study region. While simulating the traffic conditions, we paid attention to control necessary variables in order to get a convincing result. Four microscopic simulation scenarios are defined in PTV VISSIM: no signal priority (which is the current situation), traditional signal priority, pre-detective signal priority and pre-detective signal priority with coordination. The simulation was carried out 1 time for every scenario. The realization of signal priority for buses is a loop of real-time information exchange. It uses Visual Basic to write an external program based on the results of theoretical studies, and calls the initialized PTV VISSIM simulation objects through the PTV VISSIM COM API for performing various tasks interface. PTV VISSIM uses single-step simulation. Its simulation resolution is 1 time step per simulation second. It acquires the simulation time of buses reaching the detectors by estimating the detectors' status at the end of each simulation second and sends the information back to an external functioning program for processing. After calculating the signal timing adjustment algorithm, the PTV VISSIM simulation model obtains a new signal timing, which is returned and applied to the PTV VISSIM simulation objects. It is through repeated cycles that the PTV VISSIM simulation acquires the effect of dynamic bus signal optimization and adjustment.

PTV VISSIM is a micro-simulation software which simulation is built on travel behaviour data. The reallife travel behaviour is embodied in the software using a set of parameters. Appropriate value for each parameter leads to nice simulation result. However, the travel behaviour of drivers from different countries varies a lot, due to the different driving habits, driving environment and driving skills they have. A slight difference in the parameter could result in a total different simulation output. In order to show more convincing results of our proposed control strategy, we need to ensure the simulation is as close as possible to the real travel condition in China, which needs necessary corrections about the travel behaviour parameters. Berkhout and Righolt (2011), based the large investigations on recent China's traffic patterns and the cooperation with Chinese scholars, has proposed a method to adjust the travel behaviour parameters in PTV VISSIM. This research is 
very meaningful to improving the simulation accuracy when we research on recent Chinese traffic. According to Berkhout's and Righolt's (2011) research results, there are obvious differences between the default value of CC2 which means car following variance and CC3 equalling car following threshold in PTV VISSIM and the real driver behaviour in China. To make them representative, we changed their values from $4 \mathrm{~m}$ and -8 to $3.45 \mathrm{~m}$ and -4.5 respectively. Other parameters are set as follows: the minimum look ahead distance is $0 \mathrm{~m}$; observed vehicles are 2; headway is $0.9 \mathrm{~s}$ and vibration acceleration is $0.25 \mathrm{~m} / \mathrm{s}^{2}$. In addition, according to the definition of transferred distance in Section 1.2, the setting of dwell time in PTV VISSIM simulation is very important. There are two dwell time models in PTV VISSIM, one follows normal distribution and the other is empirical distribution. In normal distribution, once the two parameters, mean $\mu$ and variance $\sigma$, are known, the normal distribution is also determined. The empirical distribution is based on the mathematical method of estimating probability from frequency, defined by generating different charts (usually the accumulative probability curve) from a minimal value, a maximal value and any values in between. In this research, we chose the normal distribution to match the dwell time model of buses arriving at stations. Since simulation is going to reveal real dynamic traffic operation, its data must come from real life to establish the model in PTV VISSIM. We collected buses' arrival time to stations in Changzhou during peak hours (17:30-18:30). Sample size is 215. Sample mean $\mu$ is $14.73 \mathrm{~s}$ and the sample variance $\sigma$ is $9 \mathrm{~s}$. Knowing the value of these parameters, the normal distribution is easily determined.

\section{Simulation Scenarios and Analysis}

\subsection{BRT Line 1 in Changzhou}

Changzhou, the study case in this paper, is located in the central zone of the Yangtze River delta in China. It is one of the very first cities in China to be equipped with BRT. Now it has two BRT arterial lines with a total length of $46 \mathrm{~km}$ (line 1 travels from North to South, while line 2 travels from East to West). It also has 12 BRT branch lines. The average daily passenger volume of all BRT lines in Changzhou is 327800 , accounting for $28.44 \%$ of the average daily passenger volume of the whole city.

The study area is a road section of Tongjiang south road, located in the inner city of Changzhou, shown in Fig. 3a.

The AADT of the study section has reached approximately $50000 \mathrm{pcu} / \mathrm{d}$, and it is the equivalent traffic volume including all types of vehicles traveling on Tongjiang south road. This two-way street has 6 lanes. Each lane is approximately $3.5-3.8 \mathrm{~m}$ wide. The exclusive bus lanes are set in the middle of the road, with a width of $3.5 \mathrm{~m}$ each. From Dahongqi road to Feilong road, there is a median barrier in part of the exclusive bus lanes. The study section consists of four intersections. Details of this section and its channelizing design are shown in Fig. 3b. There are two bus stations in this section: Wan Fuqiao station and Fei long station. Evening peak hour passenger flow at the two bus stations is 550 and 650 . This paper chooses the intersection of Feilong road, where two arterial roads are joined, as the main intersection due to its high traffic flow. The road section used in simulation is $1.8 \mathrm{~km}$ long. Within the section, there is a joining of an artery and a branch line, which is selected as the secondary intersection. The detecting area is from the intersection of Guanhe west road to the intersection of Feilong road. The artery line B1 and branch lines $\mathrm{B}_{6}$ and $\mathrm{B} 1_{9}$ are chosen as the study objects. $\mathrm{B} 1_{6}$ has a line of $24.5 \mathrm{~km}$ long with headway of 10 min. The line of $\mathrm{Bl}_{9}$ is $23.3 \mathrm{~km}$ long with average headway of $6 \mathrm{~min}$. The average space interval of its stations is $960 \mathrm{~m}$. In some road sections, they share the same route with $\mathrm{B} 1$. Working together with the main line B1, these two branch lines have taken a large volume of passengers in the study region. The accumulated bus service frequency is approximately $15-20$ vehicles per hour, and the total evening peak hour cross-section traffic flow of these three lines is 2567. Our study is focused on traffic from North to South, since the southbound has a very heavy traffic during the evening peak hours and it has already become a big burden to the road traffic. a)

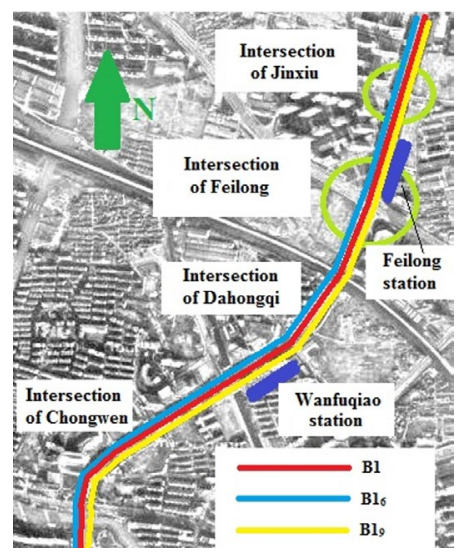

b)

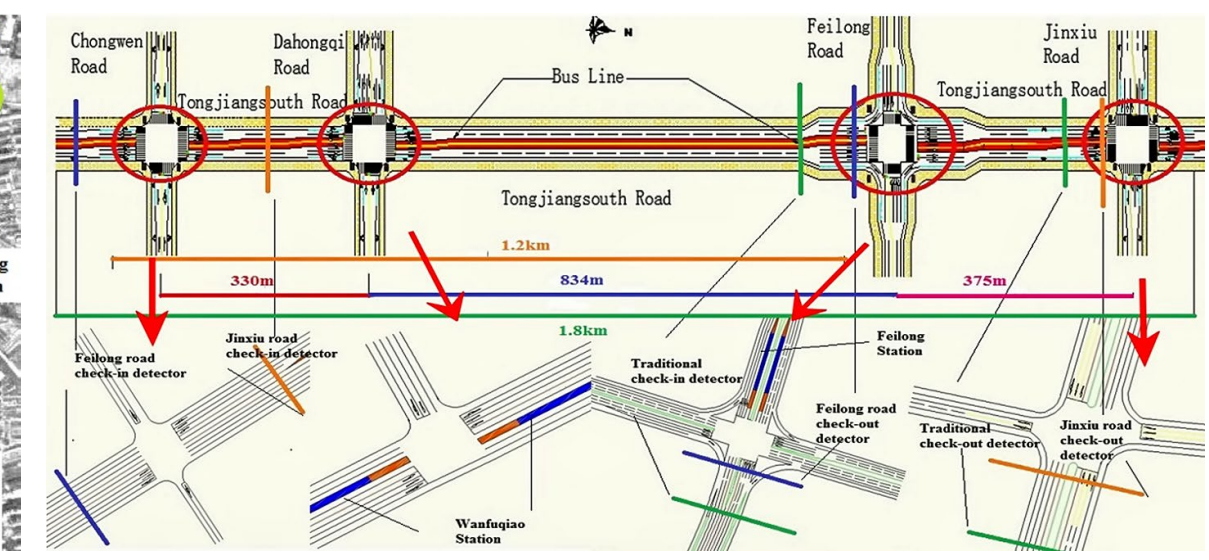

Fig. 3. Tongjiang south road in Changzhou (a) and sketch map of intersections in the study section (b) 


\subsection{Simulation Scenarios}

Simulation was done based on field survey data. According to Table 1, the parameters in simulation are close to those from the real survey data.

To evaluate the effectiveness of our control strategy, we designed four simulation scenarios. The only difference between each scenario is the control strategy, while other factors are strictly controlled.

Scenario 1: no signal priority (the current situation). This scenario involves the microscopic simulation of the existing control strategy. The signal parameters are set using the data collected from the survey.

Scenario 2: traditional signal priority for BRT. In the traditional signal priority for BRT, detectors are placed upstream and close to the stop line of the exclusive bus lane. Decisions are made either to extend of the green time or shorten the red time of the bus service phase based on the data collected by the detectors.

Scenario 3: pre-detective signal priority. Compared with Scenario 2, pre-detective signal priority extends the detecting section. In this paper, considering the specific circumstance of Tongjiang south road, and the algorithm described in Section 1.1, the detectors are placed about $1.2 \mathrm{~km}$ (equal 100-150 s in advance) upstream the intersection However, this method can only be applied to primary intersections, which means in Scenario 3, there is no intended coordination between intersections. The signal control of each intersection is mutually independent, and the adjacent intersections gained no benefit from the signal priority served for the primary intersection. In this way, Scenario 3 is designed to serve as a control group for Scenario 4 , in order to show the advantages of coordination and prove that our strategy can be effective.

Scenario 4: pre-detective signal priority with coordination. Compared with Scenario 3, this scenario offers more specific signal cycle constraints and takes the coordination between the primary and secondary intersections into consideration.

\subsection{Data Survey}

Static and dynamic data are both used in this study. The static data include road and traffic infrastructure, intersection information and bus deployment with different routes. The dynamic data consist of the traffic flow information and bus operation.

Dynamic data is analysed through the phase sequences, the lengths of green time of each phase and the traffic flow in each direction. The flow we used here is particularly collected during the evening peak, from 17:30 to $18: 30$ (Fig. 4).

Based on Changzhou's data, the simulation has successfully and abstractly represented the real condition. Here are some rules to translate the time in simulation to the real time. Since simulation starts at time '0', in real life it corresponds to 17:30:00, which is the starting time of our data collection. One second in simulation equals to one second in real life. Our data collection ends at 18:30:00, and correspondingly, the simulation lasts for 1 hour as well and end at time ' 3600 s.' The first bus of each line $\left(\mathrm{B} 1, \mathrm{~B}_{6}\right.$ and $\left.\mathrm{B} 1_{9}\right)$ appeared in the study area at the 120 th, 600th and 480th second respectively in simulation. For example, the first B1 bus entered our study section at 17:32:00, which matches with time '120 s' in simulation. Each bus line has a departure interval of $120 \mathrm{~s}, 600 \mathrm{~s}$ and $480 \mathrm{~s}$ respectively. We numbered every bus that showed up during the simulation according to their sequence to pass the detectors located at the beginning of signal priority section. During simulation, once the detector was activated, the count variable remembered it, so we recorded in the Visual Basic program the number of every bus, as well as their application for signal priority, to illustrate how the buses react to the predetective signal priority with coordination, and how well they behave under this new strategy. During the whole simulation, numbers of the buses that meet the requirements of application for priority are $1,2,4,8,10,12,14$, 16,18 and 20. Correspondingly, their travel times at the detecting section (from signal priority starting detector to signal priority ending detector) are $143 \mathrm{~s}, 226 \mathrm{~s}, 189 \mathrm{~s}$, 274 s, 219 s, 176 s, 191 s, 266 s, 188 s and 136 s. Based on these data, the signal timing plan can be optimized to complete the signal priority assignment.

\subsection{Indicators to Evaluate the Simulation}

To acquire a comprehensive understanding of the effectiveness of the pre-detective signal priority with coordination and the influence the signal priority may have on the entire traffic system this study, concerns about the possible changes in travel patterns of both buses and private vehicles, after the new signal priority strategy was adopted. This focus can be reflected in the selection of evaluation indicators. The indicators were selected after careful consideration and referring to many existed researches. The definition of delay is in accordance with the definition in the PTV VISSIM manual.

Table 1. Comparison of simulation and survey data

\begin{tabular}{lccc|ccc}
\hline \multirow{2}{*}{ Indicators } & \multicolumn{3}{c|}{ Feilong intersection } & \multicolumn{3}{c}{ Jinxiu intersection } \\
\cline { 2 - 8 } & Survey data & $\begin{array}{c}\text { Simulation } \\
\text { data }\end{array}$ & $\begin{array}{c}\text { Simulation } \\
\text { error [\%] }\end{array}$ & Survey data & $\begin{array}{c}\text { Simulation } \\
\text { data }\end{array}$ & $\begin{array}{c}\text { Simulation } \\
\text { error [\%] }\end{array}$ \\
\hline Average delay of private traffic $[\mathrm{s}]$ & 39.3 & 41.2 & 4.61 & 16.8 & 16.3 & 2.98 \\
\hline Average delay of buses [s] & 16.7 & 17.6 & 5.39 & 17.7 & 18 & 1.69 \\
\hline Average queue length of private traffic $[\mathrm{m}]$ & 234 & 246 & 5.13 & 97 & 102 & 4.90 \\
\hline
\end{tabular}



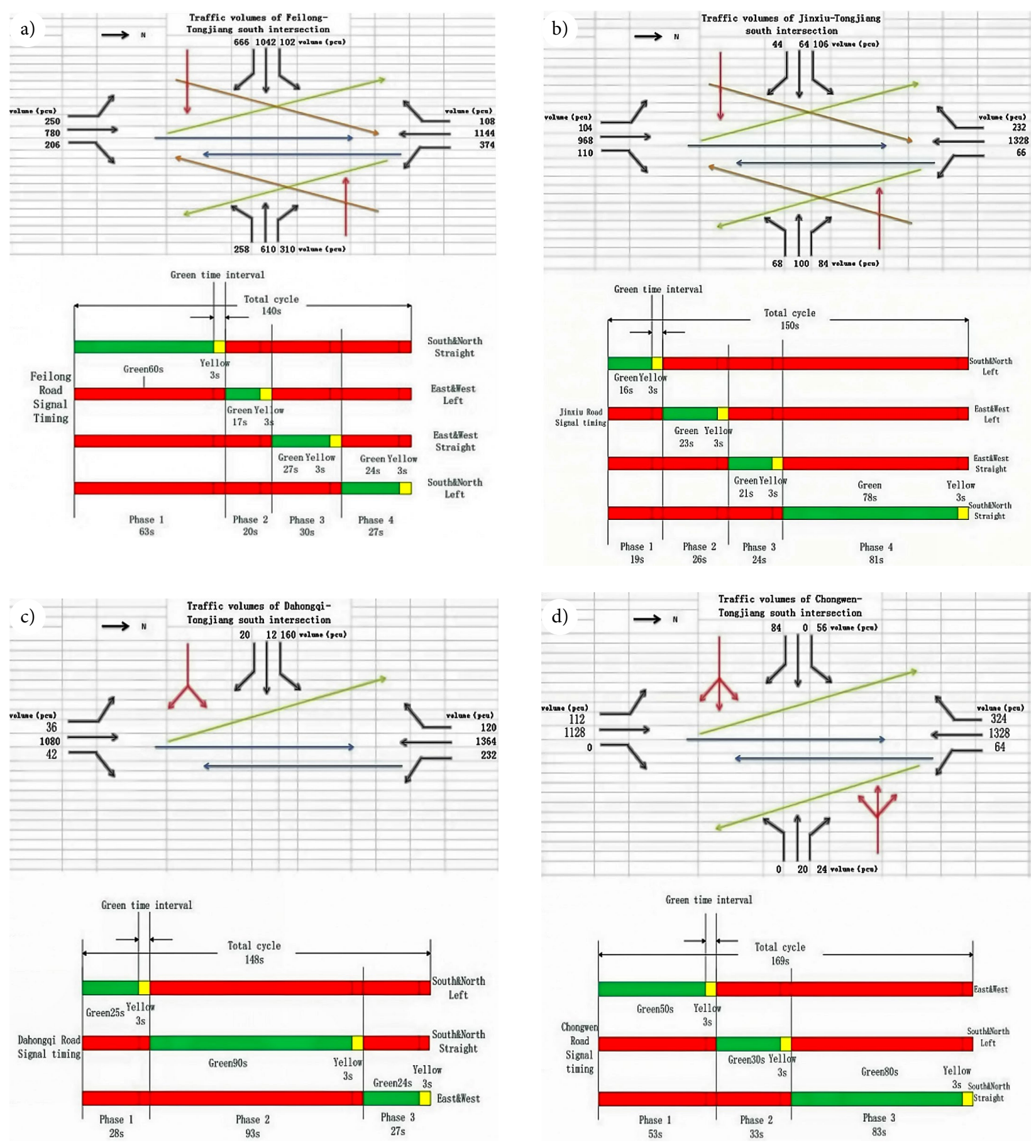

Fig. 4. Volumes and signal timings of intersections: a - Feilong road; b - Jinxiu road; c - Dahongqi road; d - Chongwen road

\subsubsection{Evaluation Indicators for Buses}

As mentioned previously, around half of the bus delay in the BRT system comes from intersections. Bus reliability is a key indicator to evaluate the quality of bus operation. It includes the stability and homogeneous of the time interval of buses arriving at stations. To be specific, we use headway adherence defined in Eq. (3) (expressed as the standard deviation of the headway of the buses reaching the station divided by expectation) to indicate this 'reliability'. The bus service reliability largely depends on the predictability of the time buses arriving at stations. The smaller the headway adherence is, the more homogeneous distribution of headway of buses arriving at stations is, meaning the arriving of the buses is more predictable. In such situation, buses operation is in a good state, and passengers would benefit. It is also the optimal results that any traffic signal planner would like to see research, which carried-out by Chen, W. and Chen, Z. (2009). Therefore, bus intersection delay and the bus station headway adherence are chosen as the evaluation indicators.

Bus intersection delay. Bus intersection delay is generated when buses are driving into the intersection 
approach. The delay can be calculated by the following formula:

$$
D_{b u s}=T_{a c t}-T_{0} \text {, }
$$

where: $D_{b u s}$ stands for the bus intersection delay; $T_{a c t}$ is the actual travel time needed by buses to pass through the intersection approach; $T_{0}$ is the theoretical travel time without any slowing down.

Headway adherence. Reliability of the bus service largely depends on the regularity of buses' arrival at stations. The bus station dwell delay may increase if there are too many buses arriving at the same station at the same time, especially during peak hours. Additionally, this delay may cascade into further bad effects, such as making buses arriving late at the stations downstream, forcing passengers to wait even longer and the bus would become overcrowded as well. Headway adherence is chosen to evaluate the reliability of bus service in this study. Headway adherence refers to the ratio of the standard deviation of the arrival time interval of a certain bus line in a certain period of time to the average arrival time interval of this bus line in the same period of time:

$$
\operatorname{Cov}(h)=\frac{\sigma(h)}{E(h)},
$$

where: $\operatorname{Cov}(h)$ stands for the headway adherence; $\sigma(h)$ represents the deviation of bus headway arriving at station; $E(h)$ is the average bus arrival headway (Chen, W., Chen, Z. 2009).

\subsubsection{Evaluation Indicators for Private Traffic}

In this paper, the evaluation indicators for private traffic are intersection delay and intersection queue length.

Intersection delay. Intersection delay is defined by Eq. (4). In this study, intersection delay is mainly used to analyse influences on private traffic traveling through the intersection, as caused by the altered signal timing after the adoption of signal priority for buses:

$$
D_{c a r}=T_{c a r}-T_{0} \text {, }
$$

where: $D_{c a r}$ stands for private traffic intersection delay; $T_{c a r}$ is the actual travel time needed by private traffic to pass through the intersection; $T_{0}$ is the theoretical travel time without any slowing down.

Intersection queue length. Intersection queue length at the intersection is an important evaluation indicator for designing the intersection signal timing design. If the queue at the intersection is too long, it may cause interference among vehicles traveling in different directions and may reduce the intersection's traffic capacity. In this study, the intersection queue length is mainly used to analyse the influences on the queuing situation of both the primary and secondary direction of private traffic, caused by the altered signal timing after giving priority to buses.

\subsection{Evaluation of the Simulation Results}

As mentioned in the introduction, there are lots of factors that can affect vehicle delay and other traffic operation indicators. The use of simulation can effectively control factors that are less related to the research topic, and highlight the influence that signal priority may have on buses and private vehicles. Therefore, we did the following analysis with the data obtained from PTV VISSIM.

\subsubsection{Evaluation of the Buses}

Intersection delay. Table 2 shows the bus delay at the primary intersection (Feilong-Tongjiang south road) and the secondary intersection (Jinxiu-Tongjiang south road) under four simulation scenarios. Under the traditional signal priority (Scenario 2), the delay reduces by $2.1 \mathrm{~s}$ at the primary intersection and increases by $0.7 \mathrm{~s}$ at the secondary intersection, compared with the results of current situation (Scenario 1). Table 2 also demonstrates that, under pre-detective signal priority (Scenario 3), delay further decreases by approximately $12.3 \%$ and $34.8 \%$ respectively at the primary and secondary intersection, compared with the traditional signal priority strategy (Scenario 2). By extending the detecting section much longer, the pre-detective signal priority strategy can guarantee enough signal conditioning cycle time, so that the adjustment can made gentler and the potential conflicts in applying for signal priority can be effectively reduced. After coordinating the primary and secondary intersections, the pre-detective signal priority strategy performs even better. The pre-detective signal priority strategy reduces the intersection delay to $8.8 \mathrm{~s}$, and after coordination, it reduces by another $35.3 \%$. Similarly, the secondary intersection delay decreases to $2.8 \mathrm{~s}$ without coordination and continues to reduce by additional $77.0 \%$ after coordination. Combining delay at the primary and secondary intersections, the pre-detective signal priority with coordination reduces the current delay from $35.6 \mathrm{~s}$ to $11.6 \mathrm{~s}$. Pre-detective signal priority with coordination not only uses the pre-detective signal priority measures but also introduces the coordination between buses, increasing the appropriate bus signal

Table 2. Average intersection delay of each bus

\begin{tabular}{lcc|cc}
\hline & Intersection delay & $\begin{array}{c}\text { Feilong-Tongiang south intersection } \\
\text { (primary intersection) }\end{array}$ & \multicolumn{2}{c}{$\begin{array}{c}\text { Jinxiu-Tongjiang south intersection } \\
\text { (primary intersection) }\end{array}$} \\
\cline { 2 - 5 } & Delay [s] & Decrease [\%] & Delay [s] & Decrease [\%] \\
\hline Current & 17.6 & 0.0 & 18 & 0.0 \\
\hline Traditional priority & 15.5 & 11.9 & 18.7 & -3.9 \\
\hline Pre-detective & 13.6 & 12.3 & 12.2 & 34.8 \\
\hline Pre-detective coordinating & 8.8 & 35.3 & 2.8 & 77.0 \\
\hline
\end{tabular}


Table 3. Headway adherence

\begin{tabular}{ccccc}
\hline Bus line & Current & Traditional priority & Pre-detective & Pre-detective coordinating \\
\hline $\mathrm{B} 1$ & 0.139 & 0.144 & 0.108 & 0.086 \\
\hline $\mathrm{B} 1_{6}$ & 0.233 & 0.233 & 0.155 & 0.138 \\
\hline $\mathrm{B} 1_{9}$ & 0.128 & 0.126 & 0.100 & 0.080 \\
\hline
\end{tabular}

phase. This coordination allows significantly reducing the bus intersection delay and the accumulated delay of intersections downstream.

Headway adherence. As illustrated in Table 3, after adopting the signal priority strategy, headway adherence at bus stations of the three bus lines reduces to different degrees. Under the traditional signal priority (Scenario 2 ), the headway adherence of $\mathrm{B}_{6}$ and $\mathrm{B} 1_{9}$ remains almost unchanged, showing that traditional signal priority has little effect on improving the reliability of bus service within this road section. However, pre-detective signal priority with coordination (Scenario 4) can effectively minimize the bus station headway adherence. Compared with no signal priority (Scenario 1), headway adherence in these three lines $\left(\mathrm{B} 1, \mathrm{~B}_{6}\right.$ and $\left.\mathrm{B} 1_{9}\right)$ decreases by $38.1 \%, 40.7 \%$ and $37.5 \%$, respectively, demonstrating that the reliability of bus service can be improved to the greatest degree when using the pre-detective signal priority with coordination. This result also responds well to the indicator of bus intersection delay.

\subsubsection{Evaluation of the Private Traffic}

Intersection delay. As shown in Table 4, compared with no signal priority (Scenario 1), the private traffic intersection delay increases under all the other three signal priority strategies to different degrees. This result indicates that the adjustment of signal timing may more or less have negative impacts on private traffic. Traditional signal priority (Scenario 2) and pre-detective signal priority (Scenario 3) increases the accumulated delay by $34.1 \%$ and $27.3 \%$ respectively, compared with no signal priority (Scenario 1). The impact of pre-detective signal priority with coordination is the smallest among the four, with private traffic delay increasing by $9.2 \%$ only. This is because the relatively fast speed of the buses is close to that of private traffic, part of the private traffic can benefit from the coordination between the primary and secondary intersections.

Intersection queue length. Adjustment of the signal cycle has different influences on the queue lengths of private traffic on different directions. Therefore, this study takes the queue lengths on both the peak (NorthSouth) and subordinate (East-West) directions of both the primary and secondary intersections to evaluate.

Fig. 5a shows that, compared with traditional priority, if the pre-detective signal priority is adopted at the intersection of Feilong road, the queue length in the peak direction increases. It is because traditional signal priority extends the green time of the bus phase (the peak direction), while pre-detective signal priority shortens the phase time for the primary straight direction. After adoption of the pre-detective signal priority with coordination, the queue lengths of the primary direction decreases to different levels. Fig. 5b shows that the queue length at the intersection of Feilong road more than doubles after the adoption of traditional signal priority because the extension in the green time of the peak direction increases the red time of the subordinate direction. Comparatively, pre-detective signal priority has less impact on the private traffic traveling in the subordinate direction of the arterial of the Feilong road intersection. Moreover, after coordination, pre-detective signal priority is even better than before. This result fully reflects the advantages of coordination. The total queue lengths at the two intersections shown in Fig. $5 \mathrm{c}$ demonstrate that all the three signal priority strategies for buses inevitably have some negative influences on private traffic. The intersection with traditional signal priority has the longest queue (increases by $48.0 \%$ from present), while the queue length under the pre-detective signal priority strategy is relatively shorter (increases by $41.7 \%$ ). However, the queue length under coordination is only $16.4 \%$ of the current queue length, which demonstrates that coordination can significantly reduce the impact on private traffic caused by providing priority for buses.

In summary, compared with the current situation and the traditional signal priority method, pre-detective signal priority can significantly reduce the bus intersection delay and increase the reliability of bus service. With coordination, pre-detective signal priority performs even better. This method not only reduces the bus intersection delay but also interferes less with private traffic.

Table 4. Average intersection delay of private traffic

\begin{tabular}{lc|c}
\hline & \multicolumn{2}{c}{ Intersection delay } \\
\cline { 2 - 3 } Scenarios & $\begin{array}{c}\text { Feilong-Tongjiang south intersection } \\
\text { (primary intersection) }\end{array}$ & $\begin{array}{c}\text { Jinxiu-Tongjiang south intersection } \\
\text { (secondary intersection) }\end{array}$ \\
\hline Current & 41.2 & 16.3 \\
\hline Traditional priority & 60.8 & 16.3 \\
\hline Pre-detective & 54.8 & 18.7 \\
\hline Pre-detective coordinating & 46.6 & 16.2 \\
\hline
\end{tabular}


a)

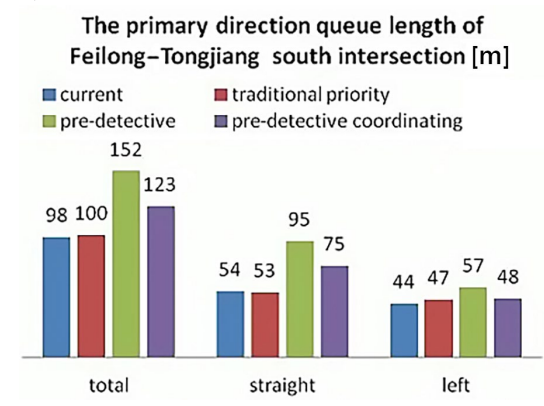

The primary direction queue length of Jinxiu-Tongjiang south intersection [m]

घcurrent $\square$ traditional priority

घpre-detective $\square$ pre-detective coordinating

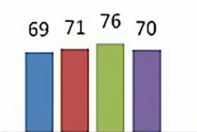

total

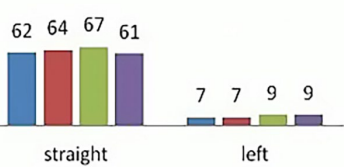

b)

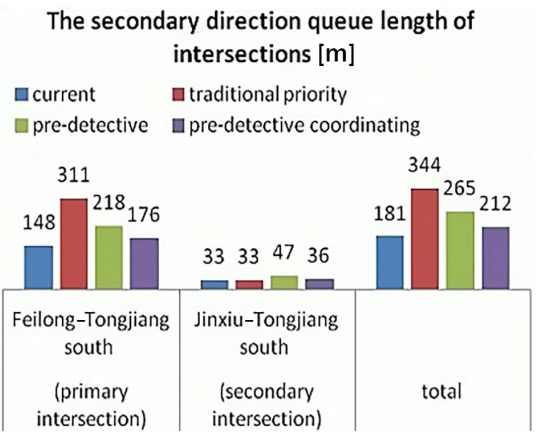

c) Total queue length of intersections [m] acurrent $\quad$ traditional priority $\square$ pre-detective $\square$ pre-detective coordinating

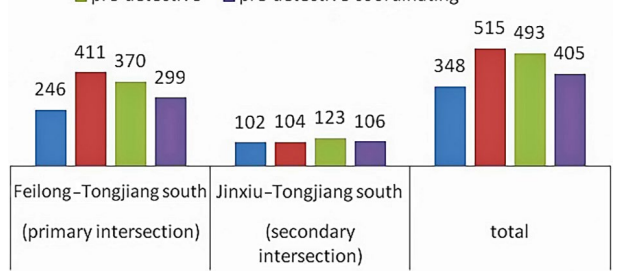

Fig. 5. Queue lengths of intersections: a - primary direction; $\mathrm{b}$ - secondary direction; $\mathrm{c}$ - total

\section{Conclusions and Future Directions}

To reduce the bus intersection delay and increase the bus operation efficiency, this study proposes a pre-detective signal priority with coordination between the primary and secondary intersections. Then, a functional module of signal priority for public transit has been developed using Visual Basic 2010 and PTV VISSIM COM. The simulation is realized in Visual Basic 2010 through PTV VISSIM COM API, when the simulation runs, data created by it comes into Visual Basic, then different signal timing will be calculated and control vehicles again. In this paper, we designed four simulation scenarios: no signal priority, traditional priority and pre-detective signal priority without and with coordination. For each scenario, a unique algorithm is designed to perform simulation in the program. The real-life data was collected from field study in Changzhou and put into the program through PTV VISSIM. With the traffic data and the four scenarios we set before, the traffic operation characteristics can be calculated and generated accordingly. To evaluate the effectiveness of each signal priority strategy, necessary indicators for both buses and private traffic are carefully chosen and defined, and the parameters and setting method required by PTV VISSIM simulation are determined. At last, this paper compares the pre-detective signal priority with coordination with the other three signal priority options according to a set of chosen indicators. The used data are from the BRT system in Changzhou, China.

Results show that, in terms of reducing bus intersection delay and increasing the reliability of bus service, pre-detective signal priority with coordination decreases the total bus intersection delay by $67.4 \%$, and the headway adherence of the 3 bus lines by approximately $40 \%$, compared with no signal priority strategy. Pre-detective signal priority with coordination performs better than the other two signal priority options. Moreover, with respect to the interferences caused to private traffic, the intersection delay only increases by $9.2 \%$ after adopting the pre-detective signal priority with coordination, compared with the no signal priority option, the value of which is much lower than the other two signal priority options. This result fully demonstrates that pre-detective signal priority with coordination can considerably reduce the intersection delay of $B R T$ and increase the reliability of BRT service. This finding also demonstrates that more determined signal cycle constraints can better guarantee the passage rights of other forms of transport. So far, the academic improvement of our study has been proved by a real case. We expect this study to serve as a reference for other cities, which are in need of increasing the reliability of urban BRT services from the perspective of signal priority for buses.

However, this study also has some deficiencies. In most Chinese cities, it is common that more than just one bus line share the same road section. The total bus frequency can be relatively high on these sections, making it even more complicated when some buses heading for different directions request for priority at the same time. To simplify this situation, this study only evaluates traffic on one direction (from South to North). Additionally, due to the multi-line operation and high departure frequency of buses, their travel time is very uncertain. Therefore, in this study, we used the measured bus travel time data without prediction. In future studies, we will evaluate the following aspects of the BRT system: how to apply the bus travel time estimated by GPS/AVL data to the pre-detective signal priority method with coordination; how to solve the conflict caused by multi-applications for priority heading for different directions appropriately at the same time; how to consider the influence of other factors, such as traffic facilities and traffic volume, travel time and weather 
conditions on the effectiveness of bus priority (though we managed to control these delay-affected variables in the simulation); and how to ensure the overall optimal efficiency of the intersection.

\section{Acknowledgements}

This research is supported by National Basic Research 973 Program (2012CB725400) and National Natural Science Foundation of China (51338003, 51378120 and 50908052).

Research Funds supported by Jiangsu Department of transportation (2011Y04) and Foundation for Young Key Teachers of Southeast University are also appreciated.

\section{References}

Berkhout, A.; Righolt, J. 2011. Chinese Driving Behavior in Nanjing Calibrating VISSIM Parameters. Project Nanjing 2009.

Chen, G. Q. 2005. Signal priority to BRT vehicles at a signalized intersection according to their headways, Computer and Communications 23(3): 11-15. (in Chinese). http://dx.doi.org/10.3963/j.issn.1674-4861.2005.03.004

Chen, W.; Chen, Z. 2009. Service reliability analysis of high frequency transit using stochastic simulation, Journal of Transportation Systems Engineering and Information Technology 9(5): 130-134.

http://dx.doi.org/10.1016/S1570-6672(08)60083-5

Eichler, M.; Daganzo, C. F. 2006. Bus lanes with intermittent priority: strategy formulae and an evaluation, Transportation Research Part B: Methodological 40(9): 731-744. http://dx.doi.org/10.1016/j.trb.2005.10.001

Ekeila, W.; Sayed, T.; El Esawey, M. 2009. Development of dynamic transit signal priority strategy, Transportation Research Record 2111: 1-9. http://dx.doi.org/10.3141/2111-01

Head, K. L. 2006. Improved Traffic Signal Priority for Transit. Transit Cooperative Research Program (TCRP) Report A-16. Transportation Research Board (TRB).

Koehler, L. A.; Kraus, W. 2010. Simultaneous control of traffic lights and bus departure for priority operation, Transportation Research Part C: Emerging Technologies 18(3): 288-298. http://dx.doi.org/10.1016/j.trc.2009.01.007

Langdon, S. M. 2002. Simulation of Houston light rail transit predictive priority operation, ITE Journal 72(11): 28-32.

Liu, H. C.; Lin, W.-H.; Tan, C.-W. 2007. Operational strategy for advanced vehicle location system-based transit signal priority, Journal of Transportation Engineering 133(9): 513-522.

http://dx.doi.org/10.1061/(ASCE)0733-947X(2007)133:9(513)

Toledo, T.; Cats, O.; Burghout, W.; Koutsopoulos, H. N. 2010. Mesoscopic simulation for transit operations, Transportation Research Part C: Emerging Technologies 18(6): 896-908. http://dx.doi.org/10.1016/j.trc.2010.02.008

TRB. 2003. Bus Rapid Transit, Volume 2: Implementation Guidelines. Transit Cooperative Research Program (TCRP) Report 90. Transportation Research Board (TRB). 233 p. Available from Internet: http://www.tcrponline.org/PDFDocuments/TCRP_RPT_90v2.pdf

Vasudevan, M. 2005. Robust Optimization Model for Bus Priority under Arterial Progression: PhD Dissertation. University of Maryland, US. 174 p.
Wadjas, Y.; Furth, P. G. 2003. Transit signal priority along arterials using advanced detection, Transportation Research Record 1856: 220-230.

http://dx.doi.org/10.3141/1856-24

Wang, T. T.; Zhang, R. H.; Zhu, X. Y.; Wu, X. G.; Zhang, R. F. 2010. Bus rapid transit in Jinan, China - applying flexibility to transit system, in TRB 89th Annual Meeting Compendium of Papers DVD, 10-14 January 2010, Washington, DC, 1-14.

Wu, G. Y.; Zhang, L. P.; Zhang, W.-B.; Tomizuka, M. 2012. Signal optimization at urban highway rail grade crossings using an online adaptive priority strategy, Journal of Transportation Engineering 138(4): 479-484. http://dx.doi.org/10.1061/(ASCE)TE.1943-5436.0000344

Xu, H. F.; Zheng, M. M. 2009. Impact of phase scheme on development and performance of a logic rule-based bus rapid transit signal priority, Journal of Transportation Engineering 135(12): 953-965. http://dx.doi.org/10.1061/(ASCE)TE.1943-5436.0000075

Yagar, S. 1993. Efficient transit priority at intersections, Transportation Research Record 1390: 10-15. 